\title{
Ce que la syntaxe nous apprend : l'exemple du pronom on dans un corpus de consultations à l'hôpital
}

\author{
Mylène Blasco ${ }^{1}$ et Paul Cappeau ${ }^{2}$ \\ ${ }^{1}$ LRL, Université Clermont Auvergne, France \\ ${ }^{2}$ FoReLL, Université de Poitiers, France
}

Résumé. Ce travail prend appui sur un corpus oral de consultations entre des patients et des médecins (DECLICS2016). L'objectif principal est de rechercher des faits linguistiques susceptibles de caractériser les participants, leur usage de la langue et les différences éventuelles entre eux. L'analyse porte sur différentes valeurs du pronom on. Il ressort de cette étude que l'on peut identifier un lien entre les emplois dégagés et les rôles sociaux des participants et que plusieurs valeurs apparaissent spécifiques de l'un ou l'autre groupe (médecins vs patients).

\begin{abstract}
This work is based on an oral corpus of consultations between patients and doctors (DECLICS2016). The main objective is to seek linguistic facts likely to characterize the participants, their use of language and possible differences between them. The analysis focuses on different values of the pronoun on. It appears from this study that a link can be identified between the jobs identified and the social roles of the participants, and that several values appear to be specific to one group or to the other group (doctors vs patients).
\end{abstract}

\section{Introduction}

Selon la façon dont ils sont constitués, les corpus (écrits et plus encore oraux) peuvent orienter les analyses (McEnery \& Wilson, 1996) vers des faits représentatifs du système de la langue (par l'accès à des données en grande quantité), ou vers des usages plus singuliers (par l'accès à des données qualitatives plus spécifiques). Dans cette deuxième perspective, divers types de variations peuvent être observés (Gadet, 2007). L'accent peut être mis sur le «genre » qui permet de préciser une tradition discursive, un cadre social d'utilisation normée du matériau linguistique (Biber, 1988 ; Maingueneau, 2004 ; Gérard, 2019). L'accent peut aussi être mis sur les usagers eux-mêmes dans le but d'établir la façon dont les locuteurs exploitent la langue et en font une utilisation différenciée (Dubar, 2007), ce que Boutet (1988) nomme «les pratiques langagières ». Notre réflexion s'inscrira dans cette dernière direction. Pour cela, nous exploiterons un ensemble de consultations médicales extraites du corpus DECLICS2016. Nous commencerons par donner quelques informations sur les données exploitées puis nous préciserons notre cadre de réflexion avant d'illustrer les exploitations différenciées de la syntaxe auxquelles se livrent les divers acteurs sociaux impliqués.

Nous souhaitons montrer qu'une analyse syntaxique portant sur un fait de langue précis (l'utilisation du pronom sujet on) apporte un éclairage précieux à la fois sur le fonctionnement de la langue et sur la posture des intervenants (Weizman, 2006). L'analyse sera conduite à deux niveaux : la description d'emplois dans des énoncés courts (afin d'établir un classement des différentes valeurs représentées) et le suivi dans sa longueur d'une interaction, qui permettra d'observer l'émergence de certaines valeurs en contexte.

\section{Présentation du corpus}

Pour une présentation détaillée du projet et du corpus DECLICS2016, nous renvoyons à Auriac-Slusarczyk \& Blasco (2019). Nous nous bornons ici à présenter la sous-partie sur laquelle portent nos analyses : les consultations. Ces dernières mettent en contact un médecin et un patient parfois accompagné d'un aidant (assez régulièrement le conjoint). Le tableau (1) précise quelques aspects quantitatifs utiles pour la suite :

\begin{tabular}{|l|l|l|l|}
\hline $\begin{array}{l}\text { Numéro du sous- } \\
\text { corpus }^{2}\end{array}$ & Cote $^{3}$ & $\begin{array}{l}\text { Nombre de mots } \\
\text { du PAT }\end{array}$ & $\begin{array}{l}\text { Nombre de mots } \\
\text { du MED }\end{array}$ \\
\hline$[\mathrm{CO} 1]$ & CO-SERVA-PAT4-MED1 & 658 & 1324 \\
\hline$[\mathrm{CO} 2]$ & CO-SERVA-PAT5-MED1 & 1886 & 1488 \\
\hline$[\mathrm{CO} 3]$ & CO-SERVA-PAT11-MED2 & 813 & 340 \\
\hline$[\mathrm{CO} 4]$ & CO-SERVA-PAT16-MED4 & 1377 & 2270 \\
\hline$[\mathrm{CO} 5]$ & CO-SERVB-PAT23-MED5 & 568 & 640 \\
\hline$[\mathrm{CO} 6]$ & CO-SERVB-PAT25-MED5 & 2081 & 1836 \\
\hline
\end{tabular}




\begin{tabular}{|l|l|l|l|}
\hline$[\mathrm{CO} 7]$ & CO-SERVB-PAT26-MED6 & 1304 & 422 \\
\hline$[\mathrm{CO} 8]$ & CO-SERVC-PAT29-MED7 & 2777 & 1473 \\
\hline$[\mathrm{CO} 9]$ & CO-SERVC-PAT33-MED8 & 1862 & 2467 \\
\hline$[\mathrm{CO} 10]$ & CO-SERVC-PAT34-MED8 & 3002 & 1405 \\
\hline$[\mathrm{CO} 11]$ & CO-SERVD-PAT2-MED9 & 3838 & 9992 \\
\cline { 2 - 5 } & TOTAL & 20166 & 23657 \\
\hline
\end{tabular}

Tableau 1. Le sous-corpus Consultations de DECLICS2016

\section{Cadre de réflexion}

Avant de présenter nos analyses, nous donnons quelques indications sur l'orientation de ce travail et rappelons succinctement quelques éléments de la description du pronom on.

\subsection{Identité, langage et rôles sociaux}

La notion d' «identité sociale » se retrouve dans de très nombreux champs disciplinaires comme l'illustre un rapide sondage dans Cairn ${ }^{4}$, ce sont en priorité les champs de la sociologie, de la psychologie, des sciences politiques, de l'histoire et de l'économie qui l'utilisent ${ }^{5}$. Les travaux de Tajfel (1972), Turner (1987), Lorenzi-Cioldi et Doise (1999), parmi beaucoup d'autres, ont permis de préciser le rôle et les fonctions de la catégorisation sociale et les incidences de la répartition des individus entre divers intergroupes (Jumageldinov, 2009).

Le déséquilibre entre la linguistique et les autres champs disciplinaires ${ }^{6}$ tient pour partie au fait que la langue fait partie des indicateurs qui relèvent de la conception que les « objectivistes » (Cuche, 1996) se font de la notion d'identité (Molina Luque, 2002). La langue n'est, dans cette optique, qu'une voie d'accès parmi bien d'autres (à côté de la religion, la psychologie collective, etc.) à cette notion. C'est pourtant par ce biais que nous nous en servirons : mettre en rapport la catégorisation sociale (et plus précisément ici un rôle ${ }^{7}$ ) avec une pratique langagière (Charaudeau, 2009 ; Véronique, 2012; Greco et al., 2014). Divers travaux récents en linguistique soulignent la part grandissante que prennent ces préoccupations (Rolland-Lozachmeur, 2013 ; Ploog et al., 2017 ; Garric \& Herbland, 2020).

Ce corpus, quoique de taille modeste, constitue un matériau précieux - constitué d'interactions écologiques - et donne accès à des échanges rarement observés ou disponibles en linguistique (Ploog et al., 2018 ; Garric \& Herbland, 2020). La constitution du corpus permet de travailler avec la notion de « rôles sociaux » (Coenen-Huther, 2005). Lors de la consultation, chaque intervenant occupe, de fait, une place particulière de patient ou de médecin (Rey \& Romain, 2018). Notre hypothèse est que ce rôle a des incidences sur le matériau langagier mobilisé. Le rôle, vu comme « une notion intermédiaire entre celles d'individu et de société » (Nadel, 1957), offre un niveau d'observation original de la variation : ni celui du genre, ni celui de l'individu ${ }^{8}$ pris comme instance isolée. L'objectif de nos analyses consiste à identifier les sous-parties du système ou les valeurs que les participants activent et à mettre à jour de possibles différences entre leurs paroles.

Pourquoi faire le choix d'une analyse de type syntaxique ? D'une part, parce qu'il s'agit d'une composante de la langue dont on est sûr qu'elle figurera dans toutes les productions. D'autre part, on sait que la syntaxe se prête à de nombreux faits de variation (Blanche-Benveniste, 1997). Enfin parce que notre analyse à deux niveaux (dans des énoncés pour observer des faits de distribution et dans des passages suivis) nous semble une voie d'accès adaptée qui offre des indications intéressantes.

\subsection{Ce que l'on sait sur on}

De très nombreux articles ont été consacrés aux différentes valeurs du pronom on, soit de façon générale en langue avec quelquefois un intérêt pour la langue parlée (Blanche-Benveniste, 2003) soit de façon plus ciblée, avec une visée stylistique, dans des productions particulières (Attal, 1987 ; Détrie, 1998) et plus récemment dans sa fonction discursive (Landragin \& Tanguy, 2014). Les multiples « facettes » (Fløttum et al., 2007) de ce pronom expliquent probablement l'intérêt qu'il suscite; la diversité des approches ainsi que les multiples cadres théoriques invoqués ne semblent pas épuiser le sujet.

Les études signalent la malléabilité du pronom (plusieurs articles utilisent l'image du « caméléon » pour caractériser ce pronom) qui peut ou non inclure le locuteur. Les classements proposés par les auteurs diffèrent notamment :

- par le nombre de rubriques : Viollet (1988) identifie 4 valeurs référentielles de on, Guerin (2006) en utilise 6 tout comme Fløttum et al. (2007) même si elles ne se recouvrent pas totalement 
- par les dénominations : Viollet (1988) utilise « une valeur parcours », Guerin (2006) parle de " renvoi à tout le monde », Fløttum et al. (2007) recourent à une double désignation (l'ensemble référentiel visé et les autres pronoms correspondants).

Ces différences sont en partie liées aux corpus utilisés qui jouent un rôle important dans l'établissement des typologies. Malgré tout, la compatibilité entre les classements des auteurs reste forte. Parmi les emplois les plus souvent repérés, on pourra signaler :

- une valeur en lien avec le locuteur (emploi dit inclusif),

- une autre exclusive (parce qu'elle n'intègre pas le locuteur)

- et une dernière souvent qualifiée d'indéfinie (Viollet parle de « locuteur universel »).

Nous nous contenterons de ces 3 valeurs générales qui permettent de proposer un classement et de soulever des questions en lien avec nos données.

La question du rapport entre nous et on est abordée par Blanche-Benveniste (1987) à travers la composition de chacun de ces pronoms. Dans les deux cas il s'agit de formes dites " complexes » en ce sens qu'elles peuvent inclure des personnes différentes. La formule utilisée pour nous ( + moi \pm toi \pm lui) indique que le moi fait partie intégrante du pronom nous. La formule décrivant le on ( \pm moi \pm toi \pm lui) n'impose pas la présence du moi. Cette différence de composition se vérifie au travers des restrictions de combinaisons syntagmatiques. Un pronom complexe en position sujet ne peut coexister avec l'une de ses composantes en position complément. Il en découle que nous + me ne sera pas attesté (* nous me parlons), alors que on + me est possible (on me parle) dès lors que le on n'est pas inclusif.

Notons enfin que Fløttum et al. (2007) permettent de faire le lien entre les deux aspects de notre cadre de réflexion (i.e. la question de l'identité et la forme linguistique observée). En effet, comme ils l'écrivent :

le pronom on, notamment en contraste avec d'autres représentations de personnes, contribue de façon décisive dans la construction de l'identité des participants de l'interaction. (p. 76)

\section{L'utilisation du pronom on dans le corpus DECLICS2016}

Dans cette partie, nous présentons d'abord quelques indications chiffrées concernant les consultations. Les deux points suivants précisent les emplois rencontrés dans les paroles des médecins et des patients. Enfin une rapide synthèse est fournie afin de faire ressortir les spécificités les plus frappantes.

\subsection{L'emploi de on dans le corpus (observation quantitative)}

Ces distinctions semblent une entrée adaptée à l'examen des valeurs du on durant les consultations : les locuteurs parlent-ils en leur nom propre et à quelle occasion recourent-ils à l'emploi du on ?

On peut d'emblée noter que l'usage du on est nettement plus important chez les médecins et que, sans surprise, le je est largement dominant chez les patients (tableau 2) :

\begin{tabular}{|c|c|c|c|}
\hline & PAT & MED & Écart \\
\hline Nombre de mots & 20166 & 23657 & $+17 \%$ \\
\hline Nombre de on & 153 & 350 & $+128 \%$ \\
\hline Nombre de $j e / j$ & 1238 & 450 & $-64 \%$ \\
\hline
\end{tabular}

Tableau 2. Emplois de on et je chez les PAT et les MED (DECLICS2016)

Si l'on regarde plus précisément chaque sous-corpus (Graphique 1), il en ressort que le pronom on :

- se rencontre chez tous les médecins (alors qu'il est absent chez un patient en CO5);

- est généralement plus abondant dans l'usage des médecins (sauf dans un cas en CO3) ;

- connaît une variation individuelle assez sensible (tant chez les médecins que chez les patients). 


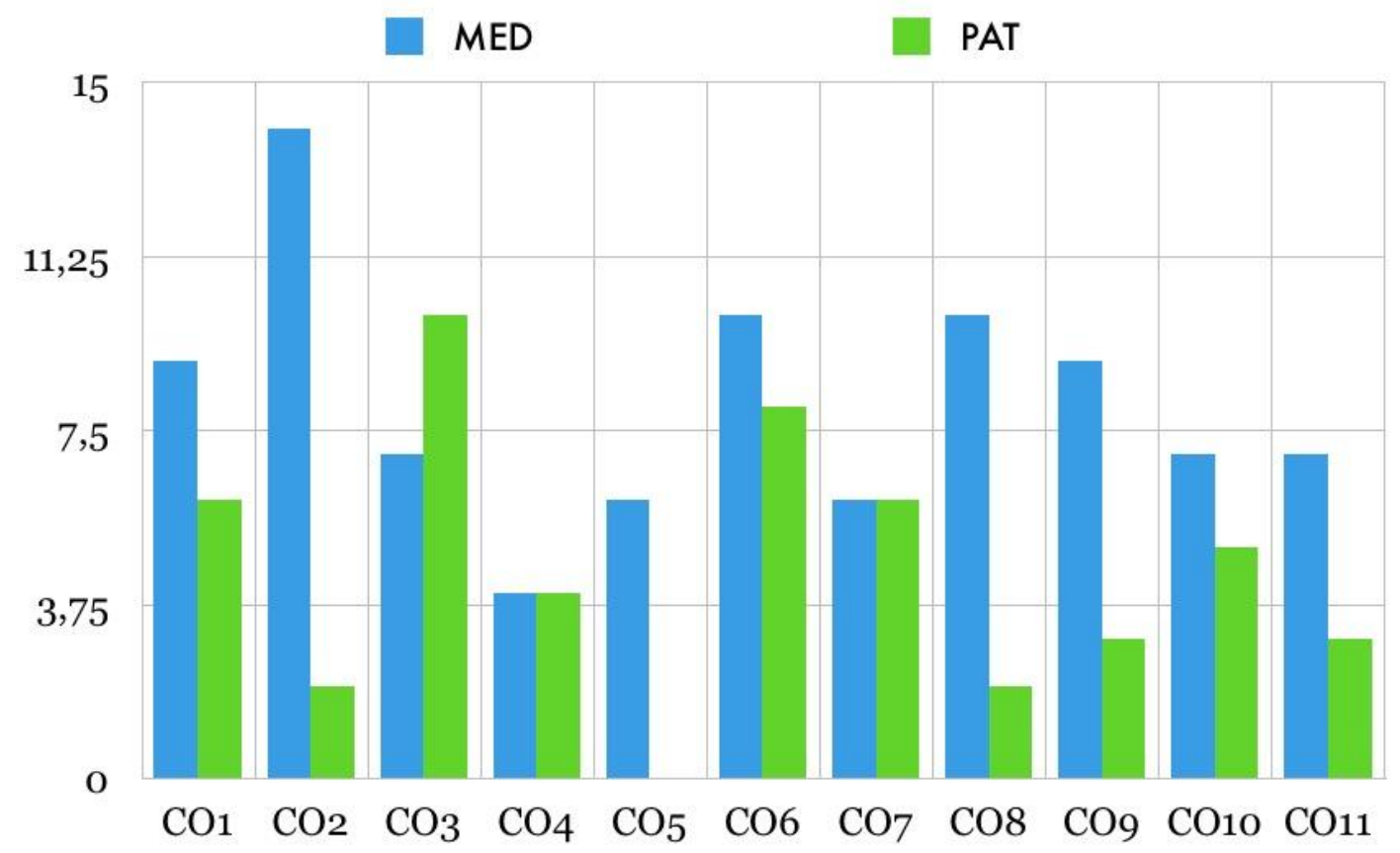

Graphique 1. Nombre d'occurrences moyen de on pour 500 mots

Les données quantitatives confortent notre choix initial d'examiner séparément l'emploi de on chez les deux types de locuteurs : médecins et patients.

\subsection{Le on chez les médecins}

On trouve dans la parole des médecins les trois valeurs du on déjà bien répertoriées qui s'enrichissent de valeurs secondaires ou d'effets de sens particuliers :

a) une valeur inclusive (c'est-à-dire qui inclut le locuteur). Celle-ci est parfois soulignée ou renforcée par le couplage avec le pronom nous :

(1) bah écoutez je vais le noter de toute façon on \#1 va se revoir (CO9) ${ }^{9}$

(2) voilà c'est pareil nous on est sur la rupture de stock [...] on a des listes d'attente (CO8)

Lorsqu'il est question des traitements, les médecins emploient assez régulièrement le sujet on qui peut donner le sentiment d'associer le patient comme en (3), là où l'emploi du je pourrait donner l'impression d'une décision unilatérale (même si l'augmentation du dosage relève bien d'une décision du médecin) et en (4) la correction dévoile la valeur personnelle du $o n^{10}$.

(3) est-ce que depuis qu'on a augmenté le Modopar de trois à quatre + vous avez l'impression que ça a modifié euh: votre tremblement $(\mathrm{CO} 4)$

(4) ça les sueurs \# on va s'en occuper moi je vais m'occuper aujourd'hui hein (CO10)

Cette valeur peut s'élargir jusqu'à désigner / représenter le corps médical (auquel le locuteur appartient), comme l'illustre (5). Les exemples avec la tournure ce qu'on appelle (6), quand ils sont produits par le médecin, se rattachent généralement à cette valeur secondaire. Des noms présents dans l'environnement, comme service (7), viennent renforcer cette interprétation.

(5) MED: c'est-à-dire qu'on avait observé que dans certaines situations les hématies euh sédimentaient plus vite que euh

PAT: d'accord

MED: que euh dans d'autres situations + et après petit à petit on a démembré tout ça et on sait quels sont les différents composants de la V.S (CO11)

(6) sinon donc vous vous prenez des ++ des toxiques des + enfin des toxiques + ce que nous on appelle toxique cannabis des choses comme ça (CO9)

(7) et ce qu'on propose actue- ici dans le service actuellement c'est un petit peu différent quand même (CO6) 
b) une valeur exclusive qui peut correspondre à d'autres que moi (cf. la dernière occurrence dans $8^{11}$ ) ou être équivalente à vous, comme en (9), ce que la réponse du patient permet de vérifier ${ }^{12}$ ou en (10) où la correction identifie bien cette valeur :

(8) non ils voulaient qu'on que je vous voie + \#1 d'après ce qu'on m'a transmis (CO11)

(9) MED : pour le moment on (n') a pas les résultats c'est ça

PAT: bah moi il m'a $+* N O M *$ il m'a rien dit non $+(\mathrm{CO} 5)$

(10)je pense qu'on a vous avez déjà élaboré pas mal de d'angoisses + (CO10)

c) une valeur présentée comme indéfinie qui est censée englober n'importe quel être humain. C'est ce que l'on trouverait par exemple dans (11) où le locuteur précise même le sens du pronom à l'aide d'un prolongement lexical (les humains) ou dans (12) dans lequel on pourrait être glosé par chaque individu :

(11) quand + on a euh un: une agression + quelque chose à laquelle il faut faire face eh bien on a besoin de plus d'énergie pour pouvoir faire face donc on a on a besoin de plus de sucre donc le + on est programmé les humains à euh voilà pour pouvoir faire monter son taux de sucre grâce à l'hormone du stress (CO6)

(12) vous savez on est le résultat euh de de la transmission qu'on a reçue de nos parents et: de nos arrières grandsparents (CO11)

On peut toutefois se demander, au vu des exemples recensés, s'il ne serait pas opportun de distinguer des emplois généraux humains dans lesquels on désigne tout individu humain (cf. les exemples précédents) et des énoncés comme (13) dans lesquels le caractère humain du pronom s'estompe (ce qui est très surprenant) et où on semble proche de l'impersonnel $^{13}$. L'équivalence est bien meilleure avec un $\mathrm{il} / \mathrm{ce}$ ou une tournure existentielle (14) qu'avec un pronom typiquement humain ${ }^{14}$ (Cappeau, 2018) comme en (15) :

(13) a- et maintenant on a une euh nouvelle molécule donc c'est toujours un trois en un (CO8)

b-chez les gens anormaux + on a la même courbe + mais qui va être décalée + par exemple vers le haut (CO11)

c- et oui mais là je pense que là on est dans la coïncidence (CO11)

(14) a- et maintenant il y a une nouvelle molécule

b- il y a la même courbe qui va être décalée

c- c'est une coïncidence

(15) a-? quelqu'un/n'importe qui/ tout un chacun a une nouvelle molécule

b- ? quelqu'un/n'importe qui/ tout un chacun a la même courbe qui va être décalée

c-? quelqu'un/n 'importe qui/ tout un chacun est dans la cö̈ncidence

\subsection{Le on chez les patients}

Dans la parole des patients on relève les valeurs générales suivantes du on :

a) La valeur inclusive est celle qui est le plus souvent utilisée. Cet emploi est probablement favorisé par la situation puisque souvent le patient est accompagné d'un aidant lors de la consultation ou évoque sa vie familiale. La valeur du on oscille principalement entre celles de l'individuel je (16) et du collectif équivalent à nous (17). En (18), l'aidant utilise un on surprenant, puisque la valeur collective visée (nous) semble peu compatible avec le problème évoqué (aller à la selle). Mais l'emploi du on (vs un il plus conforme aux règles habituelles de la langue) souligne l'empathie de l'aidant.

(16)je me sens pas bien en fait euh enfin j-c'est voilà hein + il y a l'âge il y a le sûrement des choses qui font que:: je suis en train de prendre du poids [...] on arrive à un âge $(\mathrm{CO} 8)$

(17) + après on est tous un peu stressés dans la famille euh:: c'est:: + mon frère est pareil (CO8)

(18) AID : enfin on en prend pas tout à fait peut-être tous les jours mais \#1 bon \#

MED : \#2 ouais \# + ça je je vous conseillerais d'en prendre tout le temps hein tous les jours pour euh permettre justement à ce que vous alliez à la selle \#1 correctement \# (CO4)

De façon parallèle au médecin, le patient utilise le on quand il évoque un traitement. Chacun des participants utilise des marques linguistiques qui manifestent l'association médecin / patient. Cet emploi du pronom donne aussi parfois le sentiment d'un patient-expert, qui se pose en partenaire actif du traitement (19) : 
(19) parce qu'en fait quand je suis venu + et qu'on a mis le traitement en place $+j$ 'ai oublié l'ordonnance en fait (CO10)

Toutefois chez le patient, ce on peut en fait renvoyer à la prise de parole du médecin sous forme de discours rapporté (20) ou repris (21). Il n'est donc pas sûr que l'adhésion que le on est censé refléter soit aussi forte du côté du patient...

(20) je me souviens une des premières fois où vous êtes venue voir la stimulation vous m'avez dit peut-être qu'avec un peu de chance on enlèvera tous les médicaments $(\mathrm{CO} 2)$

(21) MED: bah non mais je me demande si vous avez pas des pas des questions par rapport à ce que j'ai écrit \{rires\}

PAT: al-à la rigueur la dis- il y a un problème aussi qu'on a du mal à poser le diagnostic c'était la dyskinésie: trachéo-bronchiale (CO11)

b) La valeur exclusive est plus souvent identifiée par la cooccurrence avec un pronom complément me de première personne (22), voir Blanche-Benveniste (1987). Cette configuration quasi absente chez les médecins (1 seul exemple, cf. (8)) est bien représentée chez les patients (17 exemples dont 7 chez un même individu).

(22) Je disais mais qu'est-ce qu'il faut que je fasse pour qu'on m'aide on me on me mettait dans mon assiette on me coupait pas ma viande (CO7)

La composition du on peut facilement fluctuer d'une occurrence à l'autre. En (23), le premier on est exclusif, le second inclusif, mais la présence d'indices (me dans le premier cas, avec monsieur $X$ dans le second lève toute ambiguïté quant à l'interprétation) :

(23)on m'avait interrompu le Liorisol en deux mille dix puis on avait décidé avec monsieur *NOM* de le reprendre l'année dernière (CO11)

c) L'emploi indéfini ne se rencontre pas dans la parole des patients. Même dans un exemple tel que (24) où le couplage avec tous oriente vers une interprétation élargie à l'ensemble de la classe, le lien avec le locuteur reste étroit et le glissement du on vers le je est effectif. Même chose avec (25) qui pourrait être vu comme un propos général mais qui précédé de dans ma famille (lors du tour de parole antérieur) restreint l'extension du on qui est alors interprété comme inclusif :

(24) + bah c'est c'est bien il faut se prévoir mais on a tous un loyer à payer + une assurance à payer + une essence à à faire le plein + je veux dire voilà on a + on a certaines responsabilités + je peux pas + non plus mettre le salaire de mon mari rien que dans mon alimentation (CO6)

(25) c'était ridicule euh on se moquait des gens des femmes qui allaient chez le coiffeur chez l'esthéticienne tout ça (CO7)

\subsection{Comparaison des emplois}

Ce relevé de différentes valeurs du on illustre que le matériau linguistique (au sens de forme + valeurs) n'est pas uniformément réparti chez les différents intervenants. Des différences importantes apparaissent lorsque l'on prend en compte les rôles sociaux, comme on le vérifie dans le tableau 3 :

\begin{tabular}{|c|c|c|}
\hline & MÉDECINS & PATIENTS \\
\hline \multirow[t]{2}{*}{ inclusif } & $\begin{array}{l}\text { - on collectif (du corps médical). Se } \\
\text { réalise notamment à travers on sait } \\
\text { (qu'il y a une des molécules, ceux qui } \\
\text { reviennent, etc.) et ce qu'on appelle } \\
\text { (des pics, le gène H.F.E., etc.) }\end{array}$ & $\begin{array}{l}\text { - on familial (le patient parle de lui et } \\
\text { de son entourage) } \\
\text { - on d'empathie (de l'aidant) qui } \\
\text { "fusionne" avec le patient }\end{array}$ \\
\hline & $\begin{array}{l}\text { - on d'adhésion (pour associer le } \\
\text { patient). Se manifeste à travers des } \\
\text { tournures comme on peut (vous aider, } \\
\text { voir un psychiatre, etc.) et on va } \\
\text { (essayer comme ça, faire ce bilan, etc.) }\end{array}$ & $\begin{array}{l}\text { - on de patient expert qui s'associe au } \\
\text { médecin pour évoquer le traitement } \\
\text { - on mimétique (quand le patient cite le } \\
\text { on d'adhésion du médecin) }\end{array}$ \\
\hline exclusif & $\begin{array}{l}\text { - pour désigner les autres : emploi rare } \\
\text { - on d'adresse au patient }\end{array}$ & $\begin{array}{l}\text { - on passif (le patient se nomme en tant } \\
\text { que patient sous le regard des autres) }\end{array}$ \\
\hline indéfini & $\begin{array}{l}\text { - on pour désigner les emplois } \\
\text { généraux humains } \\
\text { - on de distance scientifique (proche de } \\
\text { l'impersonnel) }\end{array}$ & \\
\hline
\end{tabular}


Tableau 3. Les différentes valeurs du on selon les rôles des locuteurs

Ce tableau fait apparaître le lien entre l'exploitation de la langue et le rôle social que les locuteurs remplissent. Les médecins donnent parfois l'impression d'éviter le recours au je et exploitent diverses solutions alternatives que le pronom on leur offre pour conduire l'entretien avec leur interlocuteur : associer le patient, se rattacher à un groupe (le corps médical), exploiter la valeur d'indéfini (pour se placer en position d'expert).

Lorsque les médecins emploient un pronom de première personne, il s'agit souvent d'un je factuel sorti du contexte de la consultation (26)-(27) ou d'un je d'expert, qui affirme (28) :

(26) ok + alors $+++\{$ silence, lecture dossier\} bon tout d'abord je suis désolé je pouvais pas être là vendredi + hein:: (CO11)

(27) voilà + je vais chercher la convocation je reviens pour vous donner le papier (CO2)

(28) j'ai l'expérience de de de gérer ce type de problèmes + et je sais très bien que une C.R.P à dix je vais pas bouger (CO11)

Certains passages permettent d'observer une nette distinction entre les emplois d'un on collectif (qui relie le médecin à une communauté experte) et d'un je factuel ou lié à une modalisation (29) :

(29) \#2 et on sait \# en particulier quels sont ceux qui reviennent à l'inflammation parce que c'est ce qui nous préoccupe et ceux qui reviennent à l'inflammation on les connait bien on les authentifie et j'ai bien spécifié je crois quelque part (CO11)

Les patients adoptent un positionnement bien différent, ils privilégient la composante personnelle (je) très présente même quand ils utilisent la forme on : en associant leur environnement familial (on à valeur de nous), en se reliant au médecin (bien que leur "adhésion" au traitement ne soit pas toujours totalement prise en charge). Même avec le on exclusif, ils se manifestent à travers le pronom complément me. Ils n’utilisent pas (dans le corpus) la valeur indéfinie, c'est vraiment l'expérience singulière (à travers le je) qui s'exprime.

\section{$5 \quad$ Le jeu du pronom on dans un passage suivi}

Dans cette dernière partie, nous présentons avec une mise en grille syntaxique un passage dans sa longueur pour analyser la manière dont se construit l'interaction et observer de quelles valeurs se charge le pronom on. Ici, un médecin fait le point avec le patient sur son ordonnance et l'évolution des symptômes, pour supprimer les traitements non nécessaires.

$\begin{aligned} & \text { MED : les deux croix + c'est-à-dire } \\ & \text { PAT : }\end{aligned}$ vous
$\begin{aligned} & \text { MED : } \\ & \text { PAT : }\end{aligned}$

Dans le premier passage surligné (en gris), le médecin parle en tant qu'expert, le on a une valeur collective (il permet au médecin de s'identifier au corps médical). Le lexique technique présent dans cette intervention (traite, symptômes, 
maladie, traitement) conforte ce positionnement. Le discours du médecin s'inscrit dans un protocole habituel suivi par les équipes médicales, celui-ci utilise un marqueur de justification-: on traite les symptômes parce que cette maladie il n'y a pas de traitement. Précisons que nous nous trouvons dans le cadre d'une consultation concernant une pathologie chronique, c'est-à-dire une affection qui ne se guérit pas. La seule chose à faire est de pallier les désagréments engendrés par la maladie.

Certains patients, dans le cadre d'affections non curables, sont de véritables experts de leur maladie. C'est ce qui se passe dans l'extrait. À l'amorce du médecin le Baclofène c'est pour, le patient apporte l'information attendue sous la forme d'un terme approximatif de spasticité. Puis il en donne un synonyme plus ordinaire le défaut de relâchement pour enchaîner sur un autre symptôme plus générique ou les mani-surtout les manifestations chroniques. Le patient procède à une mise en liste de troubles à laquelle le médecin acquiesce d'accord. Mais le patient poursuit en donnant un exemple précis d'une des manifestations chroniques évoquées (qu'il désigne par le pronom ça) : par exemple ça on parlait des dyskinésies trachéo-bronchiales. Dans son usage de on, le patient précise ainsi que, dans sa relation de soin avec le médecin, il entretient une forme de collaboration participative : en orangé, le on inclusif (celui le plus souvent utilisé par le patient : on parlait, on avait décidé) parle de lui et de son interlocuteur ou de lui et d'un autre médecin. Les verbes utilisés manifestent dans la relation de soin une attention portée à l'échange, au dialogue, à l'implication du patient.

Mais le patient a aussi une posture dans laquelle les décisions sont prises sans lui ou malgré lui : en bleu, le on exclusif, exprime le ressenti d'un patient plus passif (on m'avait interrompu) face à une décision dont on peut penser qu'elle ne lui appartient pas et ne lui a pas convenu (puis on avait décidé avec monsieur *NOM* de le reprendre).

Cette étude d'un passage suivi illustre bien l'utilisation qui est faite des multiples emplois du pronom on. Les différentes valeurs peuvent se succéder sans nuire à la compréhension. Ces on participent de la construction et de l'élaboration de l'interaction entre les deux locuteurs dans cette situation spécifique de consultation où ils font ensemble un état des lieux pour évaluer la prise en charge médicamenteuse et la faire progresser.

\section{Conclusion}

Chaque nouveau corpus (ou presque) offre une façon différente d'observer la langue et oriente le chercheur vers de nouvelles pistes. C'est le cas du corpus DECLICS2016 qui, par sa constitution, engage à rechercher le lien entre faits de langue et rôles sociaux. Certaines unités grammaticales s'avèrent certainement plus riches et prometteuses pour avancer dans cette perspective. Le on, parce qu'il permet d'opérer des saisies multiples, se prête bien à notre démonstration. Il a permis d'observer la répartition de certaines valeurs selon les locuteurs et de relever des éléments contextuels qui permettent d'en apprécier les subtilités. L'analyse syntaxique permet d'opérer à au moins deux niveaux (au niveau des énoncés et dans la longueur d'un échange). C'est en partie pourquoi elle constitue une entrée pertinente dans ce type de corpus et apporte un éclairage original pour comprendre quelles valeurs les locuteurs privilégient, sans peut-être s'en douter, en fonction de leur rôle. Il semble en revanche prématuré d'établir une relation orientée entre rôle et emploi des formes. La prudence conduit à ne pas trancher entre deux hypothèses : le rôle contraint-il l'emploi spécifique que le sujet parlant fait de on ou l'emploi qu'un locuteur fait de on lui permet-il de construire un rôle social spécifique ?

\section{Références bibliographiques}

Advocat, O., Blasco, M. \& Durif, F. (2019). «Les entretiens entre patients et professionnels de santé : éléments de construction collective des discours ». Revue ESASO (Education, Santé, Sociétés, Auriac-Slusarczyk, E. \& Blasco, M. (coord.), Les discours des soignants et des patients. Quelle contribution des sciences humaines et sociales?

Attal, P. (1987). Emploi de on chez La Rochefoucauld. L'information grammaticale, 32, 12-16.

Auriac-Slusarczyk, E. \& Blasco, M. (éds.) (2019). Les discours des soignants adressés aux patients à l'hôpital. Quelle contribution des sciences humaines et sociales? ». Revue ESASO (Education, Santé, Sociétés).

Bagur T. \& Portocallis G. (2017). L'individu et l'interaction, entre rôle social et identité. Revue Européenne de Coaching, 2.

Biber, D. (1988). Variation across speech and writing. Cambridge : Cambridge University Press.

Blanche-Benveniste, C. (1987). Le pronom on : propositions pour une analyse », in J.-C. Chevalier, M.-F. Delport \& M. VichCampos (éds), Les Cahiers de Fontenay, n46-48 : Mélanges offerts à Maurice Molho. Linguistique III. Fontenay-aux-Roses : ENS. 15-30.

Blanche-Benveniste, C. (1997). La notion de variation syntaxique dans la langue parlée. Langue Française, 115, 19-29.

Blanche-Benveniste, C. (2003). Le double jeu du pronom ON. In P. Hadremann, A. Van Slijcke et M. Berré (éds.). La syntaxe raisonnée, Mélanges de linguistique générale et française offerts à Annie Boone à l'occasion de son $60^{\circ}$ anniversaire. Louvainla-Neuve : De Boeck/Duculot. 43-56.

Boutet, J. (1988). La concurrence de ON et I en français parlé. Linx, 18, 47-66. 
Cappeau, P. (2018). (Pro)Noms d'humains à l'oral. Linx, 76, 57-83.

Chabrol, C. (2006). «Identités » Sociales et discursives. Questions de communication, 9, 15-27.

Charaudeau, P. (2006). Identité sociale et identité discursive, le fondement de la compétence communicationnelle. Niterói, 21, 339354.

Charaudeau, P. (2009). Identité sociale et identité discursive. Un jeu de miroir fondateur de l'activité langagière. In P. Charaudeau (dir.), Identités sociales et discursives du sujet parlant. Paris : L'Harmattan.

Coenen-Huther, J. (2005). Heurts et malheurs du concept de rôle social. Revue européenne des sciences sociales, 132. https://journals.openedition.org/ress/328

Creissels, D. (à par.) Impersonal pronouns and coreference : the case of french on. S. Manninen, K. Hietaam, E. Keiser \& V. Vihman (eds). Passives and Impersonals in European Languages. http://www.deniscreissels.fr/public/Creissels-ON.pdf

Cuche, D. (1996). La notion de culture dans les sciences sociales. Paris : La Découverte.

Détrie, C. (1998). Entre ipséité et altérité : statut énonciatif de “on” dans Sylvie. L'information grammaticale, 76, 29-33.

Dubar, C. (2007). Les sociologues face au langage et à l'individu. Langage et Société, 121-122, 29-43.

Fløttum, K., Jonasson, K. \& Norén, C. (2007). ON Pronom à facettes. Bruxelles : De Boeck.

Gadet, F. (2007). La variation sociale en français. Paris/Gap : Ophrys, (nouvelle édition).

Garric, N. \& Herbland, A. (2020). Présentation. Nouveaux discours de la santé et soin relationnel. Langage et société, 169 (1), 15-30. doi:10.3917/1s.169.0015.

Gérard, C. (2019). Linguistique des genres : objet et méthode Statut culturel des genres et variétés génériques. Linx, 78. URL : http://journals.openedition.org/linx/3030 ; DOI : 10.4000/linx.3030.

Greco, L. Mondada, L. \& Renaud, P. (dir.) (2014). Identités en interaction. Limoges : Lambert Lucas.

Guerin, E. (2006). Introduction de la notion de variation situatiolectale dans la grammaire scolaire par la caractérisation de deux opérateurs pragmatiques : on et ça. Thèse. Université de Nanterre Paris X.

Jumageldinov, A. (2009). Diversités culturelles et construction identitaire chez les jeunes appartenant aux différents groupes ethniques au Kazakhstan. Approche comparative. Thèse de doctorat. Université Lumière Lyon 2.

Landragin F. \& et Tanguy N. (2014). Référence et coréférence du pronom indéfini on. Langages, 195, 99-115.

Leeman, D. (1991). On thème. Lingvisticae Investigationes, 15-1, 101-113.

Lorenzi-Cioldi, F. \& Doise,W. (1999). Identité sociale et identité personnelle. In R. Bourhis, J.-P.H. Leyens (eds), Stéréotypes, discrimination et relations intergroupes. Liège : Mardaga.

Maingueneau, D. (2004). Retour sur une catégorie : le genre. In J.M. Adam, J-B Grize et M. ALli-Bouacha (Eds), Texte et discours : catégories pour l'analyse. Dijon : Editions Universitaires de Dijon. 107-118.

Molina Luque, F. (2002). Entre l'identité et l'identification : un problème complexe de la recherche sociologique dans le domaine de l'interculturalité. Sociétés, 76, 59-70.

Nadel, S. F. (1957). The Theory of Social Structure. Londres : Cohen and West. Tr. fr., La théorie de la structure sociale. Paris : Minuit, 1970.

Narjoux C. (2002). «On. Qui. On» ou des valeurs référentielles du pronom personnel indéfini dans Les voyageurs de l’Impériale de Louis Aragon. L'Information grammaticale, 92, 36-45.

McEnery, T. \& Wilson, A. (1996). Corpus Linguistics. Edinburgh : Edinbugh University Press.

Ploog K., Equoy-Hutin S, Mariani-Rousset S., Hezlaoui-Hamelin S., Gros- jean L. \& Pedersen L. (2017). Mécanismes de coconstruction et parcours identitaires : l'émergence de la parole dans le discours des usagers toxicomanes. In T. Guilbert \& P. Lefort (dir.), Discours et (re)constructions identitaires. Analyses interdisciplinaires, Lille : Presses universitaires du Septentrion. 159-172.

Ploog, K., Mariani-Rousset S. \& Equoy Hutin, S. (Dir.) (2018). Emmêler et démêler la parole. Approches pluridisciplinaire de la relation de soin. Besançon : Presses Universitaires de Besançon.

Rey, V. \& Romain, Ch. (2018). Les pratiques langagières au coeur de la relation de soin. In K. Ploog, S. Mariani-Rousset \& S. Equoy Hutin (Dir.). Emmêler et démêler la parole. Approches pluridisciplinaire de la relation de soin. Besançon: Presses Universitaires de Besançon, 169-185. 〈hal-01933604).

Rolland-Lozachmeur, G. (2013). Analyse énonciative des marques de la subjectivité : les relations patients/soignants/famille et l'annonce du cancer. Annales de l'université de Craiova. Filosofie IX. Craiova : Éditions Universitaria. 125-142.

Tajfel, H. (1972). La catégorisation sociale. In S. Moscovici (dir.), Introduction à la psychologie sociale. Paris : Librairie Larousse.

Turner, J-C. (1987). Rediscovering the social group : A self categorization theory. Oxford : Basil Blackwell,

Véronique, G. D. (2012). Catégorisation sociale et pratiques langagières : les analyses psychosociologiques de Paul Wald. Langage et société, 142, 11-22. 
Viollet, C. (1988). Mais qui est on ? Linx, 18, 67-75.

Weizman, E. (2006). Rôles et identités dans les interactions conflictuelles. Questions de communication, 6.

${ }^{1}$ Corresponding author : mylene.blasco-dulbecco@uca.fr - Paul.Cappeau@univ-poitiers.fr

${ }^{2}$ Pour faciliter le repérage des exemples, nous utiliserons cette seule indication.

${ }^{3}$ La côte comprend : l'indication de la situation (CO pour Consultations), l'identification du service par une lettre (A à D), une identification chiffrée du PATient et du MEDecin. Ce codage a été retenu afin de préserver au mieux l'anonymat des enregistrements.

${ }^{4}$ Cairn est un portail web qui donne accès à un certain nombre de publications récentes (revues et ouvrages) en sciences humaines et sociales. Voir : https://www.cairn.info.

${ }^{5}$ La requête «identité sociale » (effectuée le 28/04/2020) donne les résultats suivants : sociologie et société (1898), psychologie (1011), sciences politiques (890), histoire (742), économie, gestion (537), philosophie (348), sciences de 1'éducation (337), géographie (327), lettres et linguistique (276), info. communication (252), intérêt général (247), santé publique (136), droit (125), arts (115), sport et société (97), médecine (2). Toutes les discipline étiquetées dans Cairn y ont donc recours.

${ }^{6}$ Charaudeau (2006) se montre sensible à ce déséquilibre.

${ }^{7}$ Sur la différence entre identité et rôle on renvoie à Bagur et Portocallis (2017).

${ }^{8}$ La taille du corpus ne permet pas de creuser la piste de variations stylistiques propres à chaque locuteur.

${ }^{9}$ Principes adoptés pour la transcription :

$+:$ pauses

j'- : interruption, amorce

\#1 : chevauchement de parole (loc. 1)

c'est:: : allongement vocalique

monsieur *NOM* : anonymisation

${ }^{10} \mathrm{Il}$ s'agit d'un cas, finalement assez rare (voir plus loin), d'emploi du je par le médecin en lien avec une démarche qui lui incombe pleinement.

${ }^{11}$ La correction du premier on par je lève d'ailleurs une ambiguïté potentielle que ferait naître l'emploi du deuxième on qui lui est sans conteste exclusif.

${ }^{12}$ Une autre lecture de cet exemple est envisageable avec un on inclusif et une précision / rectification apportée par le patient qui emploie je.

${ }^{13}$ Narjoux (2002) identifie une valeur proche de celle-ci qu'elle commente en ces termes «l'agent ne constitue pas davantage le centre de l'énoncé et la commutation avec le tour impersonnel souligne bien la grammaticalisation de on » (p. 38). Mais, comme souvent dans les descriptions de on, les exemples utilisés comportent des prédicats étroitement liés à l'humain (on pouvait toujours biaiser, on ne pouvait résister, on ne peut pas croire...) ce qui rend moins nette la marginalisation de l'humain dans ces emplois.

${ }^{14}$ Creissels (à par.) présente une typologie extrêmement détaillée et précise des emplois indéfinis du pronom on. Il identifie des facteurs favorables à l'emploi de ce on indéfini : notamment l'importance de certains «genres » (comme ce qu'il appelle scientific style) ou les contextes discursifs (comme le fait de donner des instructions ou faire part de son expérience). 